\title{
Binary Gravitational Search based Algorithm for Optimum Siting and Sizing of DG and Shunt Capacitors in Radial Distribution Systems*
}

\author{
N. A. Khan ${ }^{1}$, S. Ghosh ${ }^{2}$, S. P. Ghoshal ${ }^{2}$ \\ ${ }^{1}$ Department of Electrical Engineering, Aliah University, Salt Lake, Kolkata, India \\ ${ }^{2}$ Department of Electrical Engineering, National Institute of Technology, Durgapur, India \\ Email: kinasim@gmail.com
}

Received March, 2013

\begin{abstract}
This paper presents a binary gravitational search algorithm (BGSA) is applied to solve the problem of optimal allotment of DG sets and Shunt capacitors in radial distribution systems. The problem is formulated as a nonlinear constrained single-objective optimization problem where the total line loss (TLL) and the total voltage deviations (TVD) are to be minimized separately by incorporating optimal placement of DG units and shunt capacitors with constraints which include limits on voltage, sizes of installed capacitors and DG. This BGSA is applied on the balanced IEEE 10 Bus distribution network and the results are compared with conventional binary particle swarm optimization.
\end{abstract}

Keywords: Normal Load Flow; Radial Distribution System; Distributed Generation; Shunt Capacitors; Binary Particle Swarm Optimization; Binary Gravitational Search Algorithm; Total line Loss; Total Voltage Deviation

\section{Introduction}

Distribution systems are usually radial in nature for operational simplicity. The Radial Distribution Systems (RDS) are fed at only one point, which is the substation. The substation receives power from the centralized generating stations through interconnected transmission network. The end users of electricity receive electrical power from the substation through RDS, which is a passive network. Hence, the power flow in the RDS is unidirectional. The high $(\mathrm{R} / \mathrm{X})$ ratio of the distribution lines results in large voltage drops, low voltage stability and power losses. Under critical loading conditions in certain industrial areas, RDS experiences sudden voltage collapse due to low value of voltage stability index at most of its nodes. The sizing and sitting of DG units and shunt capacitors in distribution systems is a very complex combinational optimization problem. This optimization problem involves not only integer and binary decision variables but also nonlinear, non-continuous, non-differential objective functions and constraints. The problems of this type are regarded as nondeterministic polynomial-time hard (NP-hard) problems, which pose computational complexities with some conventional analytical optimization techniques. In the literatures, very few papers [1-5] use the optimization of voltage profile as objective functions the integration of DG and shunt capacitor placement certain heuristic methods [1-5] have been reported in the literature for obtaining promising results. Recently, Rashedi et al. [6] proposed a new optimization algorithm called Gravitational Search Algorithm (GSA), which has been demonstrated to be very interesting to find solutions of unimodal and multimodal functions. GSA is based on the law of attraction of masses supported by the Newtonian gravity, which says that" a particle in the universe attracts every other one with a force that is directly proportional to the product of their masses and inversely proportional to the square of the distance between them”. The original version of GSA was designed for search spaces of real valued vectors. However, many optimization problems are set in binary discrete space, such as feature selection and dimensionality reduction [7-11] data mining [12], unit commitment [13], and cell formation [14], in which it is natural to encode solutions as binary vectors. In addition, problems defined in the real space, may be considered in the binary space, too. The solution is to display real digits with some bits in the binary mode. The binary search space is considered as a hypercube in which an agent may move to nearer and farther corners of the hypercube by flipping various numbers of the bits. In the literatures, very few papers use the optimization of voltage profile as objective functions. In this work, a binary version of GSA (BGSA) [15] is utilized to decide the optimal locations of DGs and shunt capacitors to obtain an overall better voltage profile for a radial distribution system. In the binary 
version of GSA (BGSA), the outcome of these forces is converted into a probability value for each element of the binary vector, which guides whether that elements will take on the value 0 or 1 . The objective function is to minimize Total Line Loss (TLL) and maximize the lowest voltage level of the system i.e nothing but minimize Total Voltage Deviation (TVD) to reach a better voltage profile. The locations of DGs and capacitors are formulated by binary variables as decision variables in the constraints.

\section{Power Flow Solution in Radial Distribution System}

The load flow solution is carried by the following set of recursive equations (1) and (2) derived from the single line diagram as shown in Figure 1.

$$
\begin{gathered}
P_{i+1}=P_{i}-P_{L i+1}-R_{i, i+1} \cdot\left(\frac{P_{i}^{2}+Q_{i}^{2}}{\left|V_{i}\right|^{2}}\right) \\
Q_{i+1}=Q_{i}-Q_{L i+1}-X_{i, i+1} \cdot\left(\frac{P_{i}^{2}+Q_{i}^{2}}{\left|V_{i}\right|^{2}}\right)
\end{gathered}
$$

where $P_{i}$ is the real power flow into the sending end of branch $i+1$ connecting bus $i$ and bus $i+1 ; P_{L i+1}$ is real component of load at bus $i+1$; $R_{i, i+1}$ is the resistance of line section between buses $i$ and $i+1$ and $V_{i}$ is the bus voltage magnitude at bus $i . Q_{i}$ is the reactive power flow into the sending end of branch $i+1$ connecting bus $i$ and bus $i+1 ; Q_{L i+1}$ is reactive component of load at bus $i+1 ; X_{i, i+1}$ is the reactance of line section between buses $i$ and $i+1$.

The problem of DG and shunt capacitors allotment with their proper capacities is of great importance. The installation of DG and shunt capacitors at non-optimal places can result in an increase in system losses, voltage deviations and costs. Therefore, a power system planning engineer requires an efficient and fast optimization method capable of indicating the best solution for a given

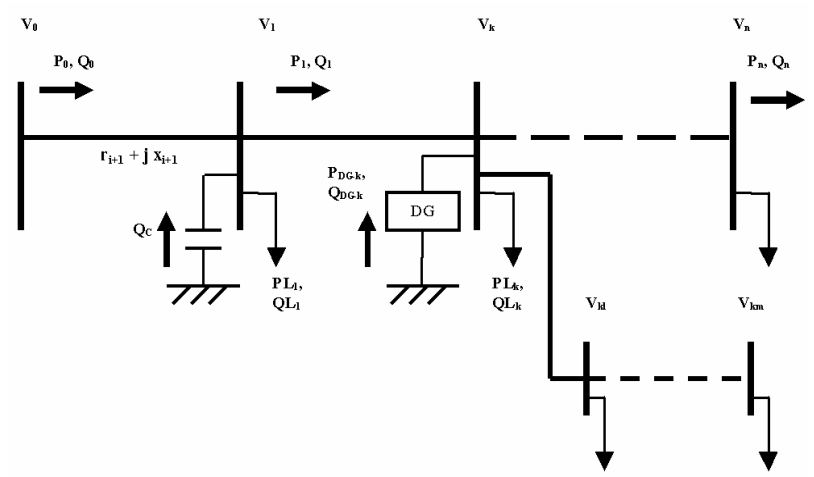

Figure 1. Single line diagram of a Radial distribution system. distribution network. The selection of the best places for installation and the preferable sizes DG and shunt capacitors banks in large distribution systems is a complex discrete optimization problem.

In order to incorporate the proposed method recursive equations (1) and (2) are modified as follows:

a) Real Power Flow with installation of DG

$$
P_{i+1}=P_{i}-P_{L i+1}-R_{i, i+1} \cdot \frac{P_{i}^{2}+Q_{i}^{2}}{\left|V_{i}\right|^{2}}+A P_{i+1} \cdot \mu P
$$

where $A P_{i+1}$ is real power magnitude injected at bus $i+1 ; \mu P$ is real power multiplier, set to zero when there is no real power source or set to 1 when there is DG power source;

b) Reactive Power Flow with shunt capacitors placement

$$
Q_{i+1}=Q_{i}-Q_{L i+1}-X_{i, i+1} \cdot \frac{P_{i}^{2}+Q_{i}^{2}}{\left|V_{i}\right|^{2}}+R P_{i+1} \cdot \mu Q
$$

where $R P_{i+1}$ is reactive capacitor power magnitude injected at bus $i+1 ; \mu Q$ is reactive capacitor power multiplier, set to zero when there is no capacitor power source or set to 1 when there is a capacitor power source;

c) Computation of Bus Voltages

$$
\begin{aligned}
V_{i+1}^{2}= & V_{i}^{2}-2\left(R_{i, i+1} \cdot P_{i}+X_{i, i+1} \cdot Q_{i}\right) \\
& +\left(R_{i, i+1}^{2}+X_{i, i+1}^{2}\right) * \frac{P_{i}^{2}+Q_{i}^{2}}{\left|V_{i}\right|^{2}}
\end{aligned}
$$

\section{Problem Formulation}

The following sections describe the details of the proposed problem formulation.

a) The Objective Functions

The main advantages of Shunt capacitors in the distribution system are loss minimization in the feeders and the improvement in the voltage profile, i.e. maintaining the voltages at customer terminals with reactive power compensation.

The following functions are computed using the proposed algorithm: Total Line Loss (TLL), Total Voltage Deviation (TVD).

b) Total Line Loss (TLL)

The installation capacitor banks should not result in an increase in the system losses. The power loss of the line section connecting buses $i$ and $i+1$ is computed as:

$$
\begin{gathered}
P_{\text {loss }}(i, i+1)=R_{i, i+1} * \frac{P_{i}^{2}+Q_{i}^{2}}{\left|V_{i}\right|^{2}} \\
T L L=\sum_{i}^{n-1} P_{\text {loss }}(i, i+1)
\end{gathered}
$$

c) Total Voltage Deviation (TVD) 
Voltage deviation can also be minimized with integration of Shunt capacitors. The total voltage deviation (TVD) in the system, which is to be minimized, is expressed as:

$$
T V D=\sum_{i 1}^{n-1}\left|1-V_{i}\right|
$$

where $i=1,2,3 \ldots \ldots n$ and $V_{i}$ is the voltage of $i^{\text {th }}$ bus in per unit for the system buses; the ideal magnitude of each bus voltage is unity.

d) Constraints

The following constraints are considered [16].

i) Total Power Conservation:

The algebraic summation of all incoming and outgoing powers over the feeders, taking into consideration the feeders' losses and the powers supplied by Shunt capacitors should be equal to the total demand at that bus.

ii) Distribution Feeder's Thermal Capacity:

Power flows in feeders must be within their capacities.

iii) Distribution Substation's Capacity:

The summation of total powers delivered to the network by the substation's transformers must be within the substation's capacity limit.

iv) Shunt capacitor Operation Limits:

The Shunt capacitor's generated power must be within the Shunt capacitor's capacity.

v) Voltage Drop Limits:

The voltage levels at different buses must be within predetermined values.

\section{Proposed Binary Gravitational Search Algorithm}

The conventional GSA was originally designed to solve problems in continuous valued space [6]. The search algorithm is based on the metaphor of gravitational interaction between masses in the Newton theory. A j-th bit of the $\mathrm{i}$-th agent $\left(x_{i j}\right)$ in a system is represented as a bit 0 or 1 where a combination of bits gives the i-th agent position.

The next agent's velocity $\left(v_{i j}\right)$ is calculated based on its current velocity and its acceleration as expressed in (9). Then, a new agent's position $\left(x_{i j}\right)$ is updated using a condition as shown in (11). However, the velocity is limited in interval $[-6,6]$ so as to achieve a good convergence rate.

$$
\begin{gathered}
v_{i j}(t+1)=r^{*} v_{i j}(t)+a_{i j}(t) \\
\text { Sigmoid }\left(v_{i}^{d}\right)=\frac{1}{1+e^{-v_{i}^{d}}} \\
x_{i}^{d}=\left\{\begin{array}{c}
0, \text { if } r>=\text { sigmoid }\left(v_{i}^{d}\right) \\
1, \text { otherwise }
\end{array}\right.
\end{gathered}
$$

\section{Simulation Results and Discussions}

To demonstrate the performance of the proposed BGSA in solving the optimal DG and shunt capacitor placement problem, the IEEE 10 bus distribution system is used in this study. In this paper, for this particular test system, Total Line Loss (TLL) and Total Voltage Deviation (TVD) were minimized and compared to the conventional BPSO as to illustrate its performance in solving the same problem. All the optimization parameters are standardized where population size and maximum population are set to 60 and 100, respectively. In the BPSO, two positive coefficients are set to $2(c 1=c 2=2)$ and inertia weight, $(w)$ monotonously decreases from $0.9\left(w_{\max }\right)$ to $0.4\left(w_{\min }\right)$. In the BGSA, the initial gravity constant, G0 is set to 100 and the best applying force, $\left(K_{\text {best }}\right)$ is monotonously decreased from $100 \%\left(K_{\text {best } \max }\right)$ to $2.5 \%$ ( $\left.K_{\text {bestmin }}\right)$.The proposed BGSA algorithm has been implemented on IEEE 10-bus radial distribution network.

\section{a) Test System-I}

IEEE 10 Bus [17] is a single line main feeder (Base Voltage $=23 \mathrm{KV}$, Base MVA=100 MVA) without laterals and sublaterals having total active and reactive powers of $12.368 \mathrm{MW}$ and 8.372 MVAR, respectively. Without any injection of DG active powers and Shunt capacitors' reactive powers, the normal load flow yields Total Line Loss (TLL) and total voltage deviation (TVD) as $78.3712 \mathrm{KW}$ and 0.6989 p.u., respectively.

b) Total Line Loss (TLL) minimization

Figure 2(a) represents voltage profile of IEEE 10 bus radial distribution system obtained by different optimization techniques (BPSO, BGSA) and normal power flow (NPF). TLL is improved more in the case of BGSA (87.67\%) as that of BPSO (81\%) over NPF, it can be seen from Table 1. It is observed, voltage profile is improved as that of BPSO, lowest bus voltage increased $6.38 \%$ by BPSO whereas in Binary GSA, it is improved by $8.69 \%$ as shown in Table 3 . Convergence characteristic is depicted in Figure 2(b). Loss and corresponding total voltage deviation is more reduced than that of BPSO as observed from Figures 2(c) and (d) in TLL minimization.

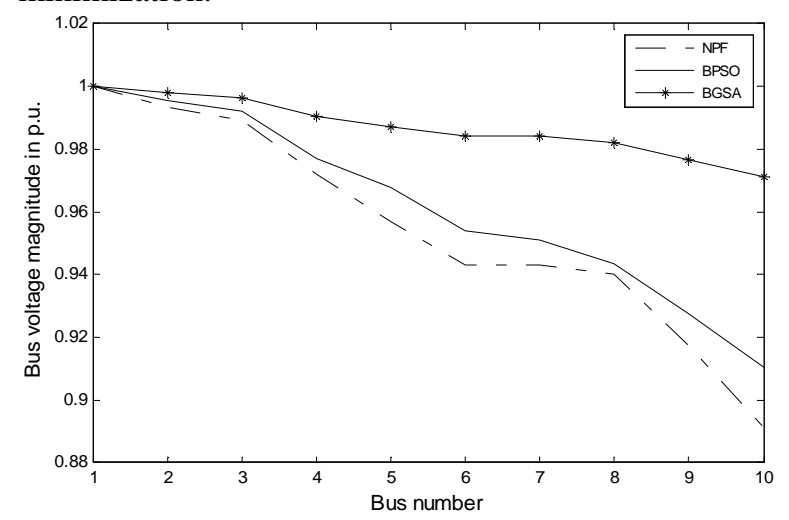

(a) 


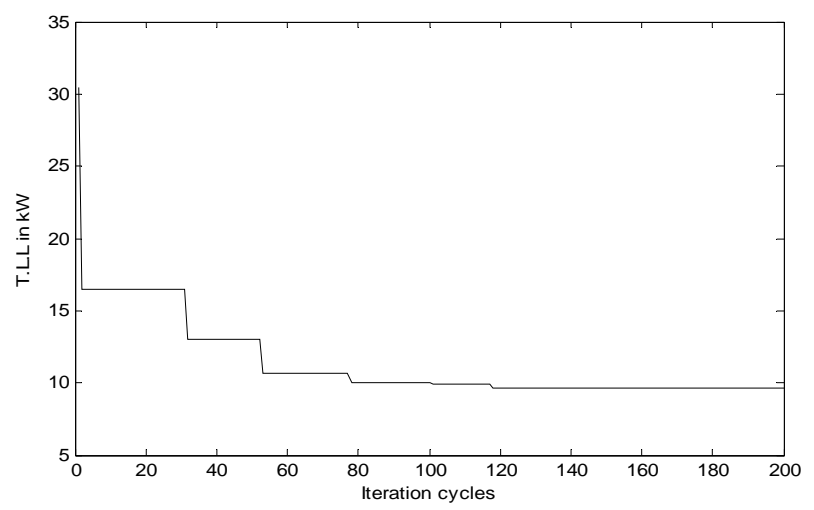

(b)

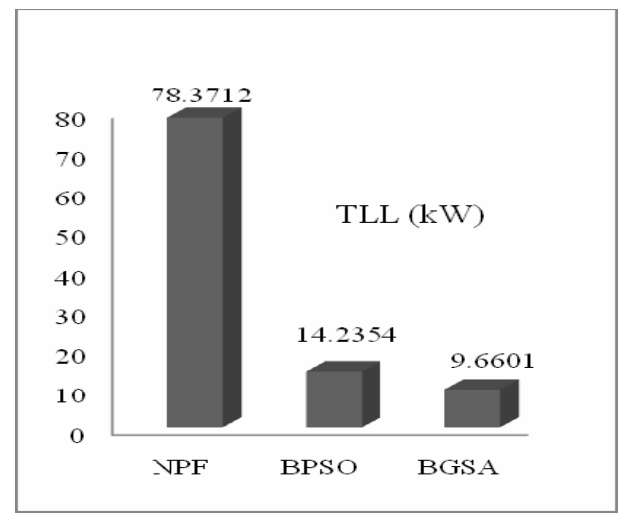

(c)

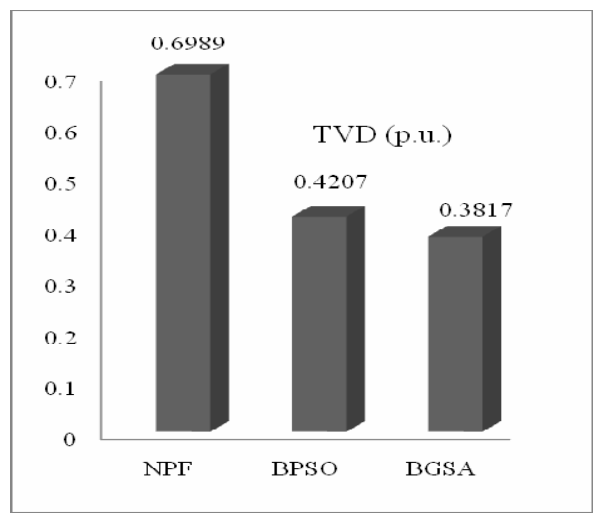

(d)

Figure 2. TLL Minimization Characteristics of IEEE 10 Bus Radial Distribution System; (a) Voltage profile obtained by different algorithms, (b) Convergence characteristic, (c) Comparison of TLL, (d) Comparison of TVD.

Table 1. Comparative study of Tll minimization of Ieee $\mathbf{1 0}$ bus test system.

\begin{tabular}{ccc}
\hline \multirow{2}{*}{ Test System } & \multicolumn{2}{c}{ Comparative Study Of TLL Minimization } \\
\cline { 2 - 3 } & $\begin{array}{c}\text { \% Improvement in } \\
\text { BPSO over NPF }\end{array}$ & $\begin{array}{c}\text { \% Improvement } \\
\text { in BGSA over NPF }\end{array}$ \\
\hline $\begin{array}{c}\text { IEEE 10 Bus } \\
\text { system }\end{array}$ & 81 & 87.67 \\
\hline
\end{tabular}

From Table 2, it is observed that same capacities of DG and shunt capacitor are used for minimizing the fitness function (TLL) in this work. Two DG of $1000 \mathrm{KW}$ (at $3^{\text {rd }}$ and $7^{\text {th }}$ bus position) and two Shunt capacitors (at $2^{\text {nd }}$ and $5^{\text {th }}$ bus position), each of $400 \mathrm{KVAR}$ are optimally placed in BGSA.

c) Total Voltage Deviation (TVD) minimization

Figure 3(a) represents voltage profile of IEEE 10 bus radial distribution system obtained by optimization techniques (BPSO, BGSA) and normal power flow (NPF). It can be seen that voltage profile is improved as that of BPSO, lowest bus voltage increased $4.47 \%$ by BPSO whereas in Binary GSA, it is improved by $8.67 \%$ corresponds to Table 4. Convergence characteristic of TVD minimization is shown in Figure 3(b). Total voltage deviation and corresponding line loss is decreased than that of BPSO as observed from Figures 3(c) and (d). One DG set of $1000 \mathrm{KW}$ capacity (at 2nd bus position), and two. TVD is improved more in the case of BGSA (8.1\%) as that of BPSO (4.47\%) over NPF as presented in Table 6.

Table 2. Capacities of DG and shunt capacitors in Tll minimization of Ieee 10 bus test system.

\begin{tabular}{cc|cc|c}
\hline & \multicolumn{3}{c}{ Comparative Study by two algorithms } \\
\cline { 2 - 5 } Test System & \multicolumn{3}{c}{ BPSO } & \multicolumn{2}{c}{ BGSA } \\
\cline { 2 - 5 } & $\begin{array}{c}\text { DG } \\
(\mathrm{kW})\end{array}$ & $\begin{array}{c}\text { Shunt Capaci- } \\
\text { tors (kVAR) }\end{array}$ & $\begin{array}{c}\text { DG } \\
(\mathrm{kW})\end{array}$ & $\begin{array}{c}\text { Shunt Ca- } \\
\text { pacitors } \\
(\mathrm{kVAR})\end{array}$ \\
\hline $\begin{array}{c}\text { IEEE 10 } \\
\text { Bus system }\end{array}$ & 2000 & 800 & 2000 & 800 \\
\hline
\end{tabular}

Table 3. Lowest bus voltage improvement in Tll minimization for Ieee 10 bus system

\begin{tabular}{ccc}
\hline \multirow{2}{*}{ Test System } & \multicolumn{2}{c}{ Comparative Study Of TLL Minimization } \\
\cline { 2 - 3 } & $\begin{array}{c}\text { \% Improvement in } \\
\text { BPSO over NPF }\end{array}$ & $\begin{array}{c}\text { \% Improvement } \\
\text { in BGSA over NPF }\end{array}$ \\
\hline $\begin{array}{c}\text { IEEE 10 Bus } \\
\text { system }\end{array}$ & 6.38 & 8.69 \\
\hline
\end{tabular}

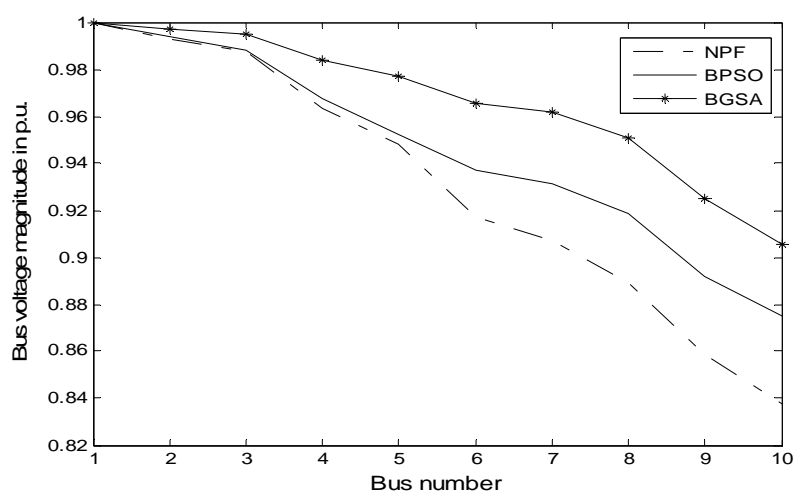

(a) 


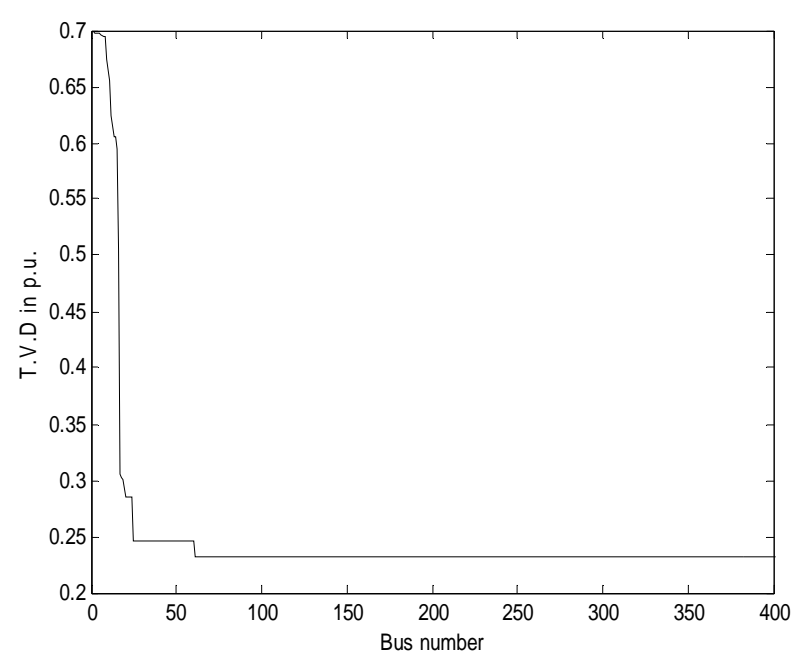

(b)

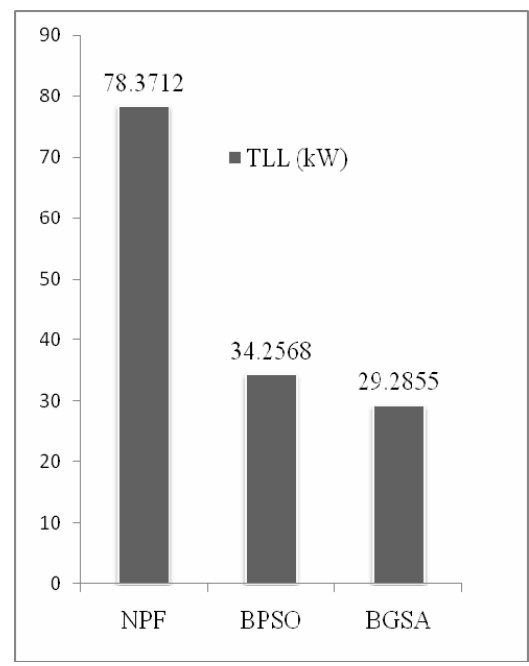

(c)

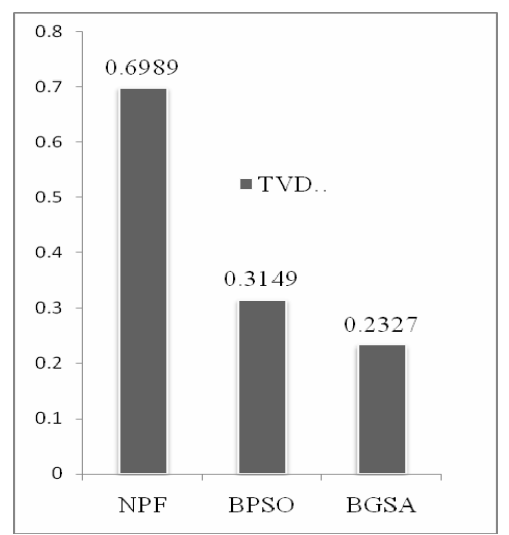

(d)

Figure 3. TVD Minimization Characteristics of IEEE 10 Bus Radial Distribution System; (a)Voltage profiles obined by different algorithms, (b) Convergence characteristic, (c) Comparison of total line loss, (d) Comparison of total voltage deviation.
Table 4. Comparative study of Tvd Minimization of Ieee 10 bus test system.

\begin{tabular}{ccc}
\hline & \multicolumn{2}{c}{ Comparative Study Of TVD Minimization } \\
\cline { 2 - 3 } Test System & $\begin{array}{c}\text { \% Improvement in } \\
\text { BPSO over NPF }\end{array}$ & $\begin{array}{c}\text { \% Improvement } \\
\text { in BGSA over NPF }\end{array}$ \\
\hline $\begin{array}{c}\text { IEEE 10 Bus } \\
\text { system }\end{array}$ & 4.47 & 13.47 \\
\hline
\end{tabular}

Table 5. Capacities of DG and shunt capacitors In TVD Minimization of Ieee 10 bus test system.

\begin{tabular}{|c|c|c|c|c|}
\hline \multirow{3}{*}{ Test System } & \multicolumn{4}{|c|}{ Comparative Study by two algorithms } \\
\hline & \multicolumn{2}{|c|}{ BPSO } & \multicolumn{2}{|c|}{ BGSA } \\
\hline & $\begin{array}{l}\text { DG } \\
(\mathrm{kW})\end{array}$ & $\begin{array}{c}\text { Shunt } \\
\text { Capacitors } \\
\text { (kVAR) }\end{array}$ & $\begin{array}{l}\text { DG } \\
(\mathrm{kW})\end{array}$ & $\begin{array}{c}\text { Shunt Ca- } \\
\text { pacitors } \\
\text { (kVAR) }\end{array}$ \\
\hline $\begin{array}{l}\text { IEEE } 10 \text { Bus } \\
\text { system }\end{array}$ & 1000 & 800 & 1000 & 800 \\
\hline
\end{tabular}

Table 6. Lowest bus voltage improvement in TVD minimization for Ieee 10 bus system.

\begin{tabular}{ccc}
\hline \multirow{2}{*}{ Test System } & \multicolumn{2}{c}{ Comparative Study Of TLL Minimization } \\
\cline { 2 - 3 } & $\begin{array}{c}\text { \% Improvement in } \\
\text { BPSO over NPF }\end{array}$ & $\begin{array}{c}\text { \% Improvement } \\
\text { in BGSA over NPF }\end{array}$ \\
\hline $\begin{array}{c}\text { IEEE 10 Bus } \\
\text { system }\end{array}$ & 4.47 & 8.10 \\
\hline
\end{tabular}

Shunt Capacitors (at 4nd and 8th bus position), each of 400 KVAR capacity, are equivalent to total 800 KVAR optimally placed in BGSA as indicated in Table 5. Same capacity of DG and Shunt capacitors are used in BPSO technique but for TVD minimization, required two DG sets and two Shunt capacitors.

\section{Conclusions}

This paper presented a BGSA and a comparative performance of BGSA and BPSO in solving the two separate single-objective optimization problem for optimal DG and Shunt capacitor placement in a radial distribution test systems. The optimization techniques have been tested on IEEE 10 bus distribution test system for determining the best optimal DG and Shunt capacitor placements for TLL and TVD minimization. The comparative results showed that the proposed BGSA is the most effective and precise among the aforementioned optimization techniques. In conclusion, the authors' contribution in this work is successful application of a binary GSA algorithm for simultaneous solution of optimal number and placements of DG powers and Shunt capacitors in a balanced distribution system. 


\section{REFERENCES}

[1] K. Zou, A. P. Agalgaonkar, K. M. Muttaqi, S. Perera. "Optimisation of Distributed Generation Units and Shunt Capacitors for Economic Operation of Distribution Systems," Power Engineering Conference; Australasian Universities; pp. 1-7, 14-17 Dec. 2008.

[2] K. Zou, A. P Agalgaonkar, K. M. Muttaqi and S. Perera, "Voltage Support by Distributed Generation Units and Shunt Capacitors in Distribution Systems," Power \& Energy Society General Meeting, IEEE, pp.1-8; 26-30 July 2009.

[3] K. Haghdar and H. A. Shayanfar, “Optimal Placement and Sizing of DG and Capacitor for the Loss Reduction via Methods of Generalized Pattern Search and Genetic Algorithm.” Power and Energy Engineering Conference Asia-Pacific, pp. 1-4, 28-31 March 2010.

[4] R. A. Hooshmand and H. Mohkami, "New Optimal Placement of Capacitors and Dispersed Generators Using Bacterial Foraging Oriented by Particle Swarm Optimization Algorithm in Distribution Systems," Springer Electr Eng 93, pp. 43-53, Jan 2011.

[5] M. Kalantari and A. Kazemi, "Placement of Distributed Generation Unit and Capacitor Allocation in Distribution Systems Using Genetic Algorithm,” pp.1-5, 8-11 May 2011.

[6] E. Rashedi, H. Nezamabadi-pour and S. Saryazdi, "Gsa: A Gravitational Search Algorithm,” Information Sciences, Vol. 179, No. 13, 2009, pp. 2232-2248. doi:10.1016/j.ins.2009.03.004

[7] P. Avishek and J. Maiti, "Development of a Hybrid Methodology for Dimensionality Reduction in Mahalanobis-Taguchi System Using Mahalanobis Distance and Binary Particle Swarm Optimization,” Expert Syst Appl Vol. 37, No. 2, 2010, pp. 1286-1293. doi:10.1016/j.eswa.2009.06.011

[8] M. Beretaa and T. Burczynski, “Comparing Binary and Real-valued Coding in Hybrid Immune Algorithm for
Feature Selection and Classification of ECG Signals.” Eng Appl Artif Intell, Vol. 20, 2007, pp. 571-585.

[9] X. Wang and J. Yang, "Feature Selection Based on Rough Sets and Particle Swarm Optimization,” Pattern Recogn Lett, Vol. 28, 2007, pp. 459-471.

[10] L. H. Chuang and H. W. Chang, "Improved Binary PSO for Feature Selection Using Gene Expression Data," Comput Biol Chem,Vol. 32, No. 1, 2008, pp.29-38.

[11] X. P. Zeng and Y. M Li, “A Dynamic Chain-like Agent Genetic Algorithm for Global Numerical Optimization and Feature Selection,” Neurocomputing, Vol. 72, 2009, pp. 214-1228. doi:10.1016/j.neucom.2008.02.010

[12] K. G Srinivasa and K. R Venugopal, “A Self-adaptive Migration Model Genetic Algorithm for Data Mining Applications. Inf Sci, Vol. 177, No. 20, 2007, pp. 4295-4313.

[13] X. Yuan and Nie, "An Improved Binary Particle Swarm Optimization for Unit Commitment Problem," Expert Syst Appl, Vol. 36, No. 4, 2009, pp. 8049-8055. doi:10.1016/j.eswa.2008.10.047

[14] T. H. Wu and C. C. Chang, "A Simulated Annealing Algorithm for Manufacturing Cell Formation Problems," Expert Syst Appl , Vol. 34, No. 3, 2008, pp. 1609-1617.

[15] E. Rashedi, H. Nezamabadi-pour and S. Saryazdi, "Bgsa: Binary Gravitational Search Algorithm,” Natural Computing, Vol. 9, 2010, pp. 727-745. doi:10.1007/s11047-009-9175-3

[16] R. Annaluru, S. Das and A. Pahwa, "Multi-level Ant Colony Algorithm for Oplacement of Capacitors in Distribution Systems," Congress on Evolutionary Computation, Vol. 2, 2004, pp. 1932-1937.

[17] J. J Grainger and S. H Lee, “Capacity Release by Shunt Capacitor Placement on Distribution Feeders, A New Voltage-Dependent Model," Power Engineering Review, IEEE, Vol. 2, No. 5, 1982, pp. 42-43. 